\title{
LA VISIÓN DEL ACUERDO DE PAZ SOBRE EL ABASTECIMIENTO DE AGUA EN EL MEDIO RURAL
}

\section{THE VISION OF THE PEACE AGREEMENT ON WATER SUPPLY IN THE RURAL ENVIRONMENT}

\author{
Andrés Gómez-Rey \\ Universidad del Rosario (Bogotá D.C., Colômbia)
}

Recebimento: 20 fev. 2017

Aceitação: 11 ago. 2017

\begin{abstract}
Como citar este artigo / How to cite this article (informe a data atual de acesso / inform the current date of access):
\end{abstract}
GÓMEZ-REY, Andrés. La visión del Acuerdo de Paz sobre el abastecimiento de agua en el medio rural. Revista da Faculdade de Direito UFPR, Curitiba, PR, Brasil, v. 62, n. 2, p. 245 - 261, maio/ago. 2017. ISSN 2236-7284. Disponível em: <http://revistas.ufpr.br/direito/article/view/50793>. Acesso em: 28 ago. 2017. DOI: http://dx.doi.org/10.5380/rfdufpr.v62i2.50793.

\section{RESUMEN}

El presente artículo pretende analizar si el acuerdo de paz suscrito el 26 de agosto de 2016 entre el Gobierno Nacional y las Fuerzas Armadas Revolucionarias de Colombia - Ejército del Pueblo (FARC-EP), así como su reformulación en la versión del 12 de noviembre de 2016 - los textos son similares, en donde únicamente cambia la inclusión de unas mayúsculas y la eliminación de la expresión "equidad de género" -, contribuye a la concreción del derecho humano al agua y representa una oportunidad en la mejora de su garantía en las zonas rurales del país, entendiendo la paz como un medio para la garantía de derechos.

\section{PALABRAS CLAVE}

Agua. Derecho humano al agua. Acuerdo de paz. Medio rural.

\begin{abstract}
This article aims to analyze whether the peace agreement signed on August 26, 2016 between the National Government and the Revolutionary Armed Forces of Colombia - People's Army (FARCEP), as well as its November 12, 2016 reformulation - both texts are similar, only differing by the inclusion of some capital letters and the omission of the expression "gender equity" -, contributes to the realization of the human right to water and represents an opportunity to improve its guarantee in rural areas of the country, understanding peace as a means to guarantee rights.
\end{abstract}

\section{KEYWORDS}

Water. Human right to water. Peace agreement. Rural environment.

\section{INTRODUCCIÓN}

El objetivo de esta investigación es comprender si el Acuerdo de Paz está construido a partir de la realidad del campo colombiano (medio rural) para buscar su desarrollo y no como 
históricamente ha ocurrido, desde “el deber ser de las cosas”. Para lograrlo, se presentan a continuación los contenidos del Acuerdo de Paz, analizando si posibilitan la satisfacción de las necesidades relacionadas con el abastecimiento de agua de la zona rural y los correlativos derechos, en relación con los distritos de riego y los servicios públicos domiciliarios.

Comencemos estableciendo que por medio rural

[...] se entiende, hoy en día, como una entidad socioeconómica y un espacio geográfico, compuesto por un territorio, una población, un conjunto de asentamientos y un conjunto de instituciones públicas y privadas. Es un conjunto de regiones o zonas en las que se asientan pueblos, aldeas, pequeñas ciudades y centros regionales, espacios naturales y cultivados y en donde se desarrolla una gran diversidad de actividades como la agricultura, la industria pequeña y mediana, el comercio, los servicios, la ganadería, la pesca, la minería, el turismo y la extracción de recursos naturales (FARAH; PÉREZ, 2004).

La anterior definición propone la existencia de una zona territorial compleja, en donde su tejido social y actores son diversos, las actividades económicas variadas y existe una presencia difusa por parte del Estado. La ruralidad colombiana es importante para el país por ser la fábrica de los alimentos que son consumidos en los cascos urbanos y los centros poblados (seguridad alimentaria) (ORGANIZACIÓN DE LAS NACIONES UNIDAS PARA LA ALIMENTACIÓN Y LA AGRICULTURA, 2009) y porque contiene la biodiversidad que caracteriza al territorio colombiano (RAMÍREZ et al., 2008). Lo anterior, sin olvidar que representa el equilibrio territorial, la fuente primordial de territorios con vocación de esparcimiento, recreación, usos agroindustriales, manufactureros, entre otros.

A través de las Bases del Plan Nacional de Desarrollo (DEPARTAMENTO NACIONAL DE PLANEACIÓN - COLOMBIA, 2014, p. 376), el Gobierno Nacional ha mencionado que el campo colombiano contribuye de manera importante al desarrollo económico y social del país. En efecto, el 84,7 \% del territorio colombiano está conformado por municipios totalmente rurales, y según la Misión para la Transformación del Campo, el 30,4 \% de la población colombiana vive en zonas rurales. Por su parte, el sector agropecuario aporta en promedio 6,1 \% del Producto Interno Bruto -PIB- total y genera el 16,3 \% del empleo del país. Además, el campo cuenta con más de 42 millones de hectáreas aptas para las actividades agropecuarias y forestales y es la fuente de los recursos naturales que ofrecen ventajas comparativas para Colombia, como los hidrocarburos, los minerales y la biodiversidad.

Sin embargo, se destaca que en Colombia el 30 \% de la población rural no tiene acceso a una fuente de agua mejorada, el $31 \%$ no tiene un sistema sanitario y el 84\% carece de servicio de alcantarillado o eliminación de excretas (DEPARTAMENTO NACIONAL DE PLANEACIÓN COLOMBIA, 2014, p. 309). La situación termina siendo tan compleja que la Comisión Económica 
para América Latina y el Caribe - CEPAL, realizó un estudio sobre los bienes y servicios públicos sociales en la zona rural de Colombia, en el cual señaló, al referirse al tema de los déficits y las brechas en acceso a los servicios públicos de acueducto y alcantarillado, o que son muy altas y que no se han reducido recientemente. De acuerdo con este estudio, la cobertura de acueducto en la zona rural es de 53,3\% y en alcantarillado es de apenas 15,6\%. Asegura también que la cobertura de hogares rurales con mecanismos alternativos de eliminación de excretas, presenta una tendencia de crecimiento de 2,8\% anual que, de mantenerse, significará alcanzar cobertura del 100\% de los hogares en la zona rural en 2022 (COMISIÓN ECONÓMICA PARA AMÉRICA LATINA Y EL CARIBE, 2016, p. 30).

Lo anterior significa que el medio rural colombiano no ha sido un especial foco de atención por parte del Estado, lo que ha conllevado a la generación de dinámicas propias y a la “autogestión”1 de las necesidades básicas de sus habitantes, quienes están principalmente representados en comunidades campesinas, afrocolombianas e indígenas. Unido a lo anterior, está el hecho de que la población rural ha sido la principal víctima del conflicto armado, siendo objeto de desplazamiento, confinamiento y violación de sus derechos humanos.

Producto de la “autogestión” de sus necesidades relacionadas con el abastecimiento de agua, los habitantes del medio rural colombiano han establecido relaciones especiales en torno a los recursos naturales renovables. Entre ellas encontramos que toda forma o sistema creado por las comunidades (refiriéndonos con ello a la infraestructura), ha buscado satisfacer los "usos múltiples del agua”2 como el consumo humano y domestico ${ }^{3}$, el uso pecuario (bovinos y aves) y el riego (TARQUINO, 2010).

De otra parte, también encontramos que estas comunidades al realizar grandes esfuerzos financieros y técnicos en relación al recurso hídrico, han afianzado sus tejidos sociales, creando modelos comunitarios de abastecimiento y respetando sus conocimientos tradicionales, lo cual se traduce en construcciones populares (CORREA C., 2006, p. 11), generando sus propias reglas de conducta ${ }^{4}$.

Adicionalmente, estas comunidades rurales han tenido que asumir grandes retos para lograr la garantía de los derechos que dependen del recurso hídrico, dada la ausencia de presencia Estatal,

\footnotetext{
${ }^{1}$ Se plantea para el presente texto como autogestión, la solución autónoma a sus necesidades básicas que sin apoyo del Estado han desarrollado las comunidades que habitan el medio rural del país.

${ }^{2}$ Las principales actividades de sustento en la ruralidad son consideradas primarias al versar sobre pesca, cultivos, minería de subsistencia, productos del bosque (incluida su extracción) y otras similares.

${ }^{3}$ Tal y como lo puede ser la preparación de alimentos, aseo personal, aseo del domicilio y vestuario.

${ }^{4}$ Reglas que incluyen las conductas populares como procesos de reproche ante su inobservancia.
} 
lo cual posee dos acepciones: de una parte, superar los aspectos técnicos del abastecimiento de agua por los bajos índices de cobertura del servicio público domiciliario de acueducto (ORGANIZACIÓN MUNDIAL DE LA SALUD, 2010) y distritos de riego dada la dispersión de las viviendas (DEFENSORÍA DEL PUEBLO - COLOMBIA, 2013, p. 126) y, de otra parte, obtener y cumplir los engorrosos y en algunos casos innecesarios requisitos y exigencias normativas ${ }^{5}$ que el Estado ha diseñado para garantizar dicho abastecimiento. Retos que además han venido siendo solucionados a través de la autogestión, a pesar de los bajos niveles de escolaridad que dificultan la gestión administrativa u operativa en sus actividades (MINISTERIO DE EDUCACIÓN DE COLOMBIA, 2014, p. 20).

Superar las dificultades que poseen las comunidades rurales y lograr un desarrollo integral del campo, implica no solo que estas tengan acceso a la tierra, sino también al agua y al saneamiento básico $^{6}$. De esta forma es posible contribuir en el proceso de alcanzar una Colombia en paz y lograr el cierre de brechas sociales, económicas y territoriales como base de las transformaciones sociales que requiere el país (DEPARTAMENTO NACIONAL DE PLANEACIÓN - COLOMBIA, 2014, p. 44).

Producto de estas problemáticas, la Corte Constitucional ha señalado que "las personas que habitan en el sector rural y tienen limitados recursos económicos, tienen derecho a ser protegidas especialmente, asegurándoles que no sean 'los últimos de la fila’ en acceder al agua potable” (CORTE CONSTITUCIONAL DE COLOMBIA, 2010) ${ }^{7}$, por las deficiencias que aun subsisten en torno a la materia.

La estrategia metodológica utilizada parte del estudio de las fuentes del derecho — norma ius fundamental, ley, doctrina, jurisprudencia y en general, el ordenamiento jurídico relacionado, en observancia del aspecto sociológico de la implementación de la norma. En consecuencia, es nuestra intensión aportar al debate y realizar propuestas que permitan incidir en el camino hacia el logro de la paz.

\footnotetext{
${ }^{5}$ A manera de ejemplo, la exigencia de la micromedición en el sector de servicios públicos es compleja de cumplir ya que las aguas rurales poseen materiales particulados (como tierra) lo cual tapona el instrumento tecnológico. Situación que implica un gasto innecesario por parte de los suscriptores.

${ }^{6}$ De conformidad con lo dispuesto en el artículo 14, numeral 19 de la Ley 142 de 1994, se entiende por saneamiento básico, "las actividades propias del conjunto de los servicios domiciliarios de alcantarillado y aseo".

${ }^{7}$ Igualmente se seguirá en este aparte el documento construido por uno de los autores para la Defensoría del Pueblo sobre las "Acciones para el Impulso del Derecho Humano al Agua en Colombia. Una mirada critica al sector de agua potable y saneamiento básico”.
} 


\section{EL AGUA EN EL ACUERDO DE PAZ}

El Acuerdo de Paz suscrito el 26 de agosto de 2016 y su reformulación del 12 de noviembre 2016, incluye relacionadas con el abastecimiento de agua de las zonas rurales, poniendo de presente la existencia de una problemática a nivel nacional sobre el acceso y abastecimiento de agua. Así las cosas, hemos dividido el análisis del acceso al agua en relación con el acuerdo de paz en cuatro ítems: comenzamos con los distritos de riego; luego abordamos el tema del servicio público domiciliario de acueducto, así pasamos a los estímulos a la economía solidaria y cooperativa y, terminando con un especial planteamiento del principio de desarrollo sostenible.

\subsection{LOS DISTRITOS DE RIEGO}

Teniendo en cuenta que la demanda de agua en Colombia está representada en el sector agrícola e industrial en un $73,2 \%$ del volumen total, mientras que el consumo humano y doméstico representa el 17,8\% (INSTITUTO DE HIDROLOGÍA, METEOROLOGÍA Y ESTUDIOS AMBIENTALES DE COLOMBIA, 2015, p. 151), se vio la necesidad de elaborar una política pública que impulsara el riego de las zonas agrícolas del país. Entre 1960 y 1990 el riego creció a una media anual de 15.000 ha, con una proporción de 2 a 1; dos tercios de inversión privada y el restante de pública (DINAR; KECK, 1996, p. 1, 6).

En los años 60 fue impulsado el riego con la creación del Instituto Colombiano de la Reforma Agraria (INCORA) y en los 70’s con el Instituto Colombiano de Hidrología, Meteorología y Adecuación de Tierras (HIMAT), sin embargo, no fue sino hasta 1993, con la Ley 41ㄹ, y los Decretos reglamentarios No. 1278 y 2135, que se logró un alcance del riego en aproximadamente unas 950 mil hectáreas (DINAR; KECK, 1996, p. 1, 6). En dicha ley, se planteó la creación de distritos de riego como estrategia de Gobierno, los cuales son “un área geográfica en donde se proporciona el servicio permanente de irrigación y drenaje, mediante obras de infraestructura hidroagrícola, como vaso de almacenamiento, derivaciones directas, plantas de bombeo, pozos, canales y caminos que en su conjunto manejan el sistema” (CORPORACIÓN AUTÓNOMA REGIONAL DE CUNDINAMARCA, 2010, p. 3) que tienen como fin surtir de agua a cultivos agrícolas a cambio de un costo no tarifario sino de inversión, llamado canon ${ }^{9}$.

\footnotetext{
${ }^{8}$ Por la cual se organiza el subsector de adecuación de tierras y se establecen sus funciones.

${ }^{9}$ Entre los costos se encuentran: i) Los beneficiarios de obras públicas realizadas por el Gobierno, que deben cancelar un cargo por revalorización de la tierra. ii) Los costos de operación y mantenimiento que se traducen en un cargo fijo por el agua y uno variable por el volumen de agua suministrado.
} 
Como su nombre lo indica, el distrito de riego posee como fin la distribución para actividades agrícolas, empresariales en mayor medida, excluyendo el uso del agua para la agricultura de subsistencia y para consumo humano directo. Sin embargo, la política pública en relación al riego ha carecido del impacto necesario en los habitantes del campo, ya que la inversión en ellos se ha visto focalizada en la industria agrícola del país, más no en su población primaria.

Teniendo en cuenta estos antecedentes, este tema se trató en el Acuerdo de Paz en el titulo 1.3 “Planes Nacionales para la Reforma Rural Integral” subtitulo 1.3.1.2 “Infraestructura y adecuación de tierras” así:

Infraestructura de riego: con el propósito de impulsar la producción agrícola familiar y la
economía campesina en general, garantizando el acceso democrático y ambientalmente
sostenible al agua, el gobierno nacional creará e implementará el Plan Nacional de Riego y
Drenaje para la economía campesina, familiar y comunitaria. Para el desarrollo del Plan se
tendrán en cuenta los siguientes criterios: La promoción y aplicación de soluciones
tecnológicas apropiadas de riego y drenaje para la economía campesina, familiar y
comunitaria, de acuerdo con las particularidades de las zonas, del proyecto productivo y de
las comunidades [...] El acompañamiento a las asociaciones de usuarios y usuarias en el
diseño y formulación de los proyectos de riego y drenaje $[\ldots .$.$] La asistencia técnica y la$
promoción de las capacidades organizativas de las comunidades para garantizar el
mantenimiento, la administración y la sostenibilidad económica y ambiental de los proyectos
de riego y drenaje $[\ldots .$.

Nótese cómo, aunque estamos en el marco del abastecimiento de agua para riego, el Acuerdo de Paz pretende la creación de un plan que permita el desarrollo de la “economía campesina, familiar y comunitaria”, histórico rezago de la ruralidad colombiana. Recordemos que el factor de pobreza es creador de dinámicas especiales; de acuerdo con la Misión para el Diseño de una Estrategia para la Reducción de la Pobreza y la Desigualdad (MERPD) (RAMÍREZ, 2005) en el año 2005, el 62\% de la población rural se encontraba en estado de pobreza, lo cual presenta un panorama complejo en la nación ya que esto involucra también baja productividad y precarias condiciones de trabajo, seguridad social, educación y salud (TARQUINO, 2010, p. 14). El porcentaje restante no ostenta condiciones óptimas, sino que simplemente no se encuentra en pobreza, pero sí es una población vulnerable de otra manera, al no contar con altos niveles de escolaridad que les ofrezca grandes ventajas comparativas. Por tanto, en las condiciones actuales, es un sector que no posee posibilidades de crecimiento por fuera de su tradicional desenvolvimiento en la "sociedad moderna”.

Ahora bien, el alto grado de pobreza de la zona rural y teniendo en cuenta que "las familias más pobres desarrollan actividades productivas en su sitio de alojamiento” (TARQUINO, 2010, p. 16), esto implica que la economía campesina, por el momento, se desarrolla al interior del hogar o residencia y no por fuera de esta, por lo cual es indispensable contar con agua para su crecimiento.

Es así como, según el CENSO 2005, el 73\% de las viviendas rurales desarrollan actividades 
económicas en su hogar como bien lo pueden ser las agropecuarias. Más aun, esta economía rural, está llamada a la producción para la supervivencia propia y sistemas económicos de menor escala, como bien lo puede ser el riego de pequeños cultivos y la cría de animales como cerdos, vacas y gallinas ${ }^{10}$.

En este sentido, encontramos que el acuerdo suscrito comprende un gran avance ya que aborda la realidad de la economía campesina y solidaria, buscando así su impulso y garantizando los derechos. Recordemos que es la desigualdad el fundamento propio de nuestro conflicto.

\subsection{DEL SERVICIO PÚBLICO DOMICILIARIO DE ACUEDUCTO}

El acueducto ${ }^{11}$ es "la distribución municipal de agua apta para el consumo humano, incluida su conexión y medición”, actividad que posee una serie de reglas técnicas ${ }^{12}$ para su puesta en marcha. Entre las principales características del servicio está el hecho que la distribución debe ser de carácter municipal y que el agua sea para consumo humano, lo cual posee una doble connotación: de un lado, que el agua esté en condiciones de potabilidad o salubridad, permitiendo con ello que no afecte la salud y vida de las personas y, de otro lado, que la destinación final que se le otorgue al agua, sea la ingesta humana, excluyendo así la distribución de agua para riego, uso pecuario y otras actividades. Igualmente se requiere que el servicio sea ofrecido en forma general o masiva ${ }^{13}$, que posea un "punto terminal” denominado suscriptor o usuario y que implique como contraprestación el pago de una suma de dinero por el servicio prestado.

En este escenario se destaca que la zona rural colombiana no se enmarca fácilmente en las características del servicio antes citadas. Por ello, el Acuerdo de Paz en el punto 1.3.2.3 expresa la necesidad de elaborar un documento que coadyuve en la satisfacción de las necesidades relacionadas con el agua y los principios que deberán ser tenidos en cuenta; así:

\footnotetext{
Vivienda y agua potable: con el propósito de garantizar condiciones de vida digna a las personas que habitan en el campo, el Gobierno Nacional creará e implementará el Plan nacional de construcción y mejoramiento de la vivienda social rural. Para el desarrollo del Plan se tendrán en cuenta los siguientes criterios: [...] La promoción y aplicación de soluciones tecnológicas apropiadas (acueductos veredales y soluciones individuales) para garantizar el acceso al agua potable y el manejo de aguas residuales [...] La asistencia técnica y la promoción de las capacidades organizativas de las comunidades para garantizar el mantenimiento, la operación y la sostenibilidad de las soluciones de acceso al agua y manejo

${ }^{10}$ Véase como soporte de esta afirmación tanto el contenido del Censo en cita como TARQUINO, 2010, p. 50.

11 Según el artículo 14 numeral 14.22 de la ley 142 de 1994.

12 Principalmente contenidas en el Reglamento Técnico del Sector de Agua Potable y Saneamiento Básico (RAS 2000).

${ }^{13}$ Esto por cuanto el artículo 366 constitucional plantea que la finalidad de los servicios domiciliarios es ofrecer de forma masiva bienes o actividades que propendan por el bienestar general y el mejoramiento de la calidad de vida de la población, mediante la solución de necesidades básicas insatisfechas.
} 
de aguas residuales [...] Promover prácticas adecuadas para el uso del agua potable [...]

De la lectura del contenido del Acuerdo se puede observar el llamado de atención sobre la necesidad de contar con un plan o ruta metodológica que gire en torno a tres aspectos principales que históricamente han sido las dificultades o retos del sector en la zona rural: soluciones tecnológicas apropiadas, asistencia técnica y prácticas adecuadas para el uso del agua potable.

La consagración de las “soluciones tecnológicas apropiadas” constituiría un gran avance en la garantía del abastecimiento de agua. Empero, para el medio rural, aun siendo un llamado de atención que proviene de la imposibilidad de prestar el servicio de forma tradicional ${ }^{14}$, esto implicaría un cambio de visión en la cual se estructura el servicio público. Por ello se requeriría de una modificación normativa e institucional sobre la materia, dado que dicha prestación presenta graves dificultades debido, como se ha mencionado, a la dispersión de las viviendas y a los bajos niveles de escolaridad allí presentes.

En consecuencia, una política que comprenda el abastecimiento del agua a través de soluciones individuales o sistemas alternativos en la prestación, es una necesidad que debe atenderse para garantizar el derecho humano al agua ${ }^{15}$, lo cual implica que se debe contar con este recurso en las condiciones y cantidades suficientes para satisfacer las necesidades básicas o elementales del ser humano. Este derecho es indispensable para vivir dignamente y es condición previa para la realización de otros derechos humanos, lo cual termina reflejando una realidad innegable "sin agua, no hay vida” (GÓMEZ-REY; RODRÍGUEZ, 2013).

Para la generación de medios alternativos para el abastecimiento de agua o la prestación a través de redes (en donde sea posible) ${ }^{16}$ se requiere sin lugar a dudas de un conocimiento de la

\footnotetext{
${ }^{14}$ Es decir, que una persona jurídica realice las actividades de captación de agua, aducción, tratamiento, almacenamiento, conducción, comercialización, transporte y/o distribución de agua, mediante una red o sistemas con puntos terminales en el domicilio de las personas.

15 Siguiendo a la Corte Constitucional de Colombia (2010): “[...] el Comité de Derechos Económicos, Sociales y Culturales, en su Observación General No. 15, indicó que respecto al derecho al agua se predican ciertas obligaciones específicas como son: (i) la disponibilidad, (ii) la accesibilidad y (iii) la calidad [...] La disponibilidad hace referencia a la cantidad suficiente del líquido vital necesario para la supervivencia humana; a la regularidad en el suministro o distribución del recurso hídrico; y a la sostenibilidad del mismo.” En palabras del Comité de Derechos Económicos esta obligación implica que "el abastecimiento de agua de cada persona debe ser continuo y suficiente para los usos personales y domésticos [...]”. La accesibilidad implica que "el agua y las instalaciones y servicios de agua deben ser accesibles para todos, sin discriminación alguna, dentro de la jurisdicción del Estado Parte [...]”. La calidad significa que "[E]l agua necesaria para cada uso personal o doméstico debe ser salubre, y por lo tanto no ha de contener microorganismos o sustancias químicas o radiactivas que puedan constituir una amenaza para la salud de las personas [...]”. Véase RED INTERNACIONAL PARA LOS DERECHOS ECONÓMICOS, SOCIALES Y CULTURALES, 2001.

${ }^{16}$ Sobre este punto véase el Titulo “j” del Reglamento Técnico para el Sector de Agua Potable y Saneamiento Básico (RAS) denominado “Alternativas Tecnológicas en Agua y Saneamiento para el Sector Rural”; donde el numeral "1.1. Alcance y Campo de Aplicación” plantea la diferencia de 2 tipos de asentamientos: “i) los centros poblados o población nucleada concentrada en caseríos o conjuntos de por lo menos 20 viviendas, separadas por paredes, muros, cercas o huertas y ii) fincas y viviendas dispersas separadas por áreas cultivadas, prados, bosques, potreros, carreteras o caminos”.
} 
actividad para que se realice de manera eficiente y efectiva, lo cual es precisamente el segundo pilar del acuerdo: "la asistencia técnica” necesaria para realizar de manera correcta la tarea.

Ahora bien, el tercer pilar de este titulo expresa la necesidad de realizar prácticas adecuadas para el uso del agua potable, lo cual se enmarca en que el agua tratada sea utilizada tanto para consumo humano, como para actividades no humanas, pero de subsistencia, como bien lo puede ser las actividades agropecuarias, como por ejemplo el mantenimiento de una cabeza de ganado, de un cerdo, unas cuantas gallinas y una pequeña huerta, entre otros.

La mencionada situación comprende un gran avance al interior del régimen legal sobre la prestación de este servicio ya que este prohíbe el uso diferente al consumo humano ${ }^{17}$, lo cual en la realidad no se cumple ya que en los cascos urbanos es también utilizada para el mantenimiento estético de vehículos y otros usos alejados del legalmente establecido.

\subsection{ESTÍMULOS A LA ECONOMÍA SOLIDARIA Y COOPERATIVA}

Teniendo en cuenta lo anterior, el Acuerdo de Paz plantea además algunos estímulos a la economía solidaria y cooperativa ${ }^{18}$. Es así como el acuerdo posee un punto de vital importancia para el impulso del real y efectivo cumplimiento del derecho humano al agua: el fortalecimiento de las formas asociativas comunitarias que asumieron esta importante tarea, a través de un plan que comprende además un enfoque de género ${ }^{19}$, basado en la solidaridad y la cooperación, que promueva la capacidad de los productores de bienes y servicios rurales. Establece el acuerdo:

Estímulos a la economía solidaria y cooperativa: con el propósito de estimular diferentes formas asociativas de trabajo de o entre pequeños y medianos productores productoras, basadas en la solidaridad y la cooperación, y que promuevan ${ }^{20}$, la autonomía económica y la capacidad organizativa en especial de las mujeres rurales, y fortalezcan la capacidad de los pequeños productores y productoras de acceder a bienes y servicios, comercializar sus productos y en general mejorar sus condiciones de vida, de trabajo y de producción, el

La densidad de población promedio del país de acuerdo con el censo DANE 2005 es de 40.7 hab./km² (cerca de 10 familias $/ \mathrm{km}^{2}$ ).

${ }^{17}$ El agua para consumo humano “[E]s aquella que por cumplir las características físicas, químicas y microbiológicas, en las condiciones señaladas en el presente decreto y demás normas que la reglamenten, es apta para consumo humano. Se utiliza en bebida directa, en la preparación de alimentos o en la higiene personal.” Es decir, agua salubre que al ser ingerida por los seres humanos, no afecte de ninguna manera su salud o su vida (Artículo 2 del Decreto 1.575 de 2007).

18 Tal y como se afirmó con anterioridad, la ruralidad colombiana posee un especial tejido social en el cual las reglas construidas por las comunidades son aquellas que rigen el comportamiento del conglomerado social. Así las cosas, la economía también es permeada por esta variable y es considerada como comunitaria, solidaria y cooperativa, al comprender particularidades públicas y otras privadas.

${ }^{19}$ Aunque el presente artículo no posee como fin la exposición del enfoque de género de los acuerdos, si es interesante contemplar que esta relación entre la mujer y el agua hoy en día posee un gran desarrollo conceptual. Véase a manera de ejemplo: <www.womenforwater.org>.

${ }^{20}$ El acuerdo suscrito en agosto de 2016 contenía la siguiente expresión que fue eliminada “[...] y la cooperación, y que promuevan la equidad de género, la autonomía económica [...]” 
Gobierno Nacional creará e implementará el Plan nacional de fomento a la economía solidaria y cooperativa rural.

Para el desarrollo del Plan se tendrán en cuenta los siguientes criterios: [...] El acompañamiento, apoyo técnico y financiero a las comunidades rurales [...] en la creación y fortalecimiento de cooperativas, asociaciones y organizaciones solidarias y comunitarias [...] El apoyo con medidas diferenciales a las organizaciones comunitarias y asociaciones para contribuir a la gestión de proyectos de infraestructura y equipamiento (vías, vivienda, salud, educación, agua y saneamiento básico, riego y drenaje) [...]

Con el fin de apoyar la economía campesina y superar las problemáticas planteadas, se debe partir del conocimiento de las particularidades del medio rural para así lograr soluciones efectivas que atiendan las necesidades y expectativas de sus habitantes. A manera de ejemplo (además de los anteriormente citados, como los usos múltiples del agua, la dispersión de las viviendas, la ausencia de grados de escolaridad) veamos algunas de las problemáticas a las cuales constantemente se enfrentan estas asociaciones encargadas de realizar el abastecimiento del agua en el medio rural ${ }^{21}$ :

a) Dado los aspectos de pobreza de estos sectores, se requiere que el abastecimiento de agua sea asequible, es decir, que las personas puedan disfrutarla de acuerdo a la capacidad económica propia de estas regiones, lo cual implica condiciones de calidad del agua especiales y una estructura tarifaria acorde a las dinámicas rurales;

b) La violencia y existencia de grupos al margen de la ley que producen inseguridad, ausentamiento de la juventud, desplazamiento, tráfico de personas, de drogas, de armas y otros, impiden un libre acceso al recurso hídrico por cuanto la propiedad o tenencia de los predios de interés de las organizaciones comunitarias que distribuyen las aguas como los nacimientos y la propiedad de las redes que componen el sistema de abastecimiento, están siendo controlados por las fuerzas propias de la guerra ${ }^{22}$. Punto anterior que posee dos connotaciones: de un lado, que en caso de no avanzar en la paz del país, las asociaciones relacionadas con el agua deben acceder a ella en sectores lejanos que no estén contralados por grupos al margen de la ley y, de otro, en el caso de comenzar la construcción de la paz, se debe trabajar para evitar la restricción al acceso de este recurso.

El fortalecimiento de estas organizaciones y su empoderamiento social debe presentarse a través del desarrollo de la de gobernanza del agua que implica un sistema participativo en torno a las cuestiones rurales de dicho recurso, donde además es indispensable, para la garantía del derecho

\footnotetext{
${ }^{21}$ Esto, sin referirnos a los trámites administrativos y exigencias legales que no están siendo cumplidas por las organizaciones tales como: reporte de información al Sistema Único de Información (SUI) que administra la Superintendencia de Servicios Públicos Domiciliarios; los permisos, concesiones y autorizaciones para el uso de los recursos naturales; los reportes de calidad de agua (concertación, materialización, toma de muestras y reporte de IRCA); análisis de índices de agua no contabilizada, estudios de costos y tarifas, cumplimiento normas tributarias y laborales entre muchos otros.

${ }^{22}$ Sin olvidar, también que en algunas ocasiones los entes territoriales u otros prestadores de mayor tamaño administrativo, desean tomar posesión de los prestadores menores o pequeños prestadores.
} 
humano al agua en la ruralidad colombiana. En consecuencia, también se les debe garantizar el acceso al agua potable y el saneamiento básico, lo que implica además fomentar la estructuración de esquemas sostenibles de acceso y saneamiento, al igual que realizar inversiones en infraestructura en las zonas rurales que habitan, como se ha planteado en el Plan Nacional de Desarrollo (DEPARTAMENTO NACIONAL DE PLANEACIÓN - COLOMBIA, 2014, p. 149).

La realidad rural colombiana ha sido construida en el ordenamiento jurídico a través de un enfoque centralista, sin embargo, al no observarse las dinámicas propias de la ruralidad colombiana, no se generó un mayor bienestar a sus habitantes. Lo anterior, puede verse en el hecho de que cada régimen jurídico de abastecimiento como servicios públicos, distritos de riego y hasta el tema ambiental, más que un impulso, impone límites o dificultades al ejercicio del derecho humano agua ${ }^{23}$, por lo cual, pensar en reformular la política del campo, es una necesidad y obligación del Estado.

En consecuencia, concordamos con la CEPAL (2016, p. 8) en que la inclusión de pobladores y territorios rurales se debe lograr con propósitos y reglas públicas claras, para que la nación asegure que sus habitantes en territorios rurales tengan los mismos derechos sociales que el conjunto de la población nacional, de modo que las brechas se reduzcan hasta desaparecer, lo que permite mejores condiciones de vida de los pobladores rurales y condiciones de desarrollo para ser cada vez más autónomos.

\subsection{UN ESPECIAL PLANTEAMIENTO DEL PRINCIPIO DE DESARROLLO SOSTENIBLE}

Producto de los puntos anteriores, el Acuerdo de Paz "Hacia un Nuevo Campo Colombiano: Reforma Rural Integral”, establece el desarrollo sostenible como un principio orientador del acuerdo y su implementación, con una redacción que difiere sustancialmente de la contenida en la Comisión Mundial del Medio Ambiente y del Desarrollo de la ONU, cuyo texto lo define así: “el desarrollo sostenible como la satisfacción de las necesidades de la generación presente sin comprometer la capacidad de las generaciones futuras para satisfacer sus propias necesidades”.

Por su parte, el Acuerdo de Paz define el principio del desarrollo sostenible de la siguiente forma: “[...] es decir, es ambiental y socialmente sostenible y requiere de la protección y promoción del acceso al agua, dentro de una concepción ordenada del territorio [...]”.

Concepto que incluye de manera directa otro enfoque al dado al principio de desarrolló

\footnotetext{
${ }^{23}$ A manera de ejemplo el cobro del servicio de evaluación para la garantía del derecho humano al agua para consumo afecta la asequibilidad del mismo. El régimen de servicios públicos exige la capacidad jurídica para contratar lo cual excluye la garantía del derecho para aquellos que no la poseen.
} 
sostenible. Con este nuevo planteamiento se está buscando que la actividad de "desarrollo" comprenda dos aspectos que la califiquen: lo ambiental y lo social, presentando además una necesidad latente: hacer partícipe a la sociedad en las decisiones de desarrollo que se adopten y la protección de los recursos naturales renovables, lo cual en últimas conlleva la necesidad de avanzar en la gobernanza, para este caso, del agua.

Ahora bien, lo curioso en la consagración del principio es que se incluye como elemento principal de dicha sostenibilidad ambiental y social del desarrollo, el agua, a través de dos visiones: el agua como derecho, al establecer la necesidad de contar con su acceso ${ }^{24} \mathrm{y}$, como un criterio que permita el orden del territorio a través del mencionado recurso, constituyéndose así un principio de desarrollo sostenible “especial” para la coyuntura del país del logro y consolidación de la paz.

El acceso al agua en condiciones de disponibilidad, accesibilidad y calidad para el medio rural, conllevaría el desarrollo integral de las personas y la población rural, al permitir que posean condiciones que superen la satisfacción de sus necesidades básicas, es decir que sus preocupaciones no versen sobre agua y alimentación diaria, sino que permitan proyectarse hacia actividades diferentes tales como la educación, el desarrollo y la tecnología.

Sobre este particular las bases del Plan Nacional de Desarrollo 2014-2018 estableció que:

[...] el acceso al agua potable y saneamiento básico son factores determinantes para mejorar las condiciones de habitabilidad de las viviendas, impactar en la situación de pobreza y salud de la población e incrementar los índices de competitividad y crecimiento del país. Sin embargo, se identifican las siguientes dificultades: 1) deficientes procesos de planeación sectorial, en algunas zonas del país, que no permiten la adecuada focalización de las inversiones; 2) esquemas de prestación de los servicios de acueducto, alcantarillado y aseo, en algunos municipios, que no son sostenibles económica financiera, ambiental y socialmente, lo que impide mejorar los indicadores de cobertura, calidad y continuidad; y 3) riesgos en la prestación de los servicios de agua potable y saneamiento básico asociados a la sostenibilidad ambiental del recurso hídrico (DEPARTAMENTO NACIONAL DE PLANEACIÓN - COLOMBIA, 2014).

Ahora bien, teniendo en cuenta que la Ley 99 de 1993 establece que el proceso de desarrollo económico y social del país se orientará según los principios universales y del desarrollo sostenible, es válido preguntarse sobre las falencias de implementación que este principio está teniendo en el

\footnotetext{
${ }^{24}$ Acceso que se presenta bien sea por: a) contar con las redes del servicio público domiciliario de acueducto o b) por tener la fuente de agua en la cercanía y contar con las autorizaciones ambientales exigidas para su utilización. Sobre este punto recordemos que, para poder captar las aguas de dominio público y de esta manera adelantar acciones del servicio de acueducto o de los distritos de riego se requiere obtener ante la autoridad ambiental de: concesión de aguas superficiales que permite el uso de los depósitos y corrientes no marítimas o continentales; permiso para la construcción de obras hidráulicas que me permite la instalación de la infraestructura necesaria para la extracción de las aguas superficiales (o estar inmerso en una reglamentación de corrientes); permiso de prospección y exploración de aguas subterráneas y su consecuente concesión, que son dos autorizaciones por medio de las cuales se otorga el derecho de construir un pozo profundo y de extraer del mismo el agua que se encuentran en depósitos y corrientes subterráneas.
} 
ordenamiento jurídico interno. Lo anterior dadas las problemáticas ambientales que actualmente se presentan en el país y la especial consagración en el Acuerdo de Paz cuyo contenido es ampliado de manera significativa en el orden social.

Seguramente el cambio de visión entre el principio consagrado en la Ley 99 de de 1993 y el acuerdo de paz obedece a las necesidades y retos constantes en relación a la contaminación y degradación del recurso hídrico, lo cual ha sido reconocido por el Estado colombiano. A manera de ejemplo encontramos que el Documento CONPES 3343 de 2005 estableció que:

\begin{abstract}
Colombia enfrenta retos importantes en términos de degradación ambiental que comprometen su desarrollo económico sostenible. Estudios preliminares sobre los costos de degradación ambiental en Colombia, indican que los impactos más considerables están asociados con la contaminación del agua [...], las inadecuadas condiciones del agua, el saneamiento y la higiene [...] se ha encontrado que la degradación ambiental afecta significativamente la salud de niños y adultos mayores, la población más pobre y grupos más vulnerables (DEPARTAMENTO NACIONAL DE PLANEACIÓN - COLOMBIA, 2005, p. $1)$.
\end{abstract}

La mencionada situación de deterioro del recurso muestra la necesidad de reformular tanto las políticas existentes, como su implementación y que mejor oportunidad que el momento histórico en el que Colombia se encuentra, en relación a la revisión del Acuerdos de Paz para comenzar con un principio de desarrollo sostenible acorde a las necesidades de todos los colombianos.

Consideramos lo anterior pertinente, sobre todo si tenemos en cuenta que, los Objetivos de Desarrollo Sostenible de Naciones Unidas ${ }^{25}$, en especial el sexto objetivo , versa sobre la necesidad de lograr el acceso universal y equitativo al agua potable, a un precio asequible para todos, incluyendo el saneamiento e higiene; aumentar sustancialmente la utilización eficiente de los recursos hídricos en todos los sectores y asegurar la sostenibilidad de la extracción y el abastecimiento de agua dulce para hacer frente a la escasez de agua y reducir sustancialmente el número de personas que sufren de escasez de agua. Igualmente poner en práctica la gestión integrada de los recursos hídricos a todos los niveles, incluso mediante la cooperación transfronteriza; proteger y restablecer los ecosistemas relacionados con el agua, incluidos los bosques, las montañas, los humedales, los ríos, los acuíferos y los lagos. Así como ampliar la cooperación internacional y el apoyo prestado a los países en desarrollo para la creación de capacidad en actividades y programas relativos al agua y el saneamiento, incluidos el acopio y almacenamiento de agua, la desalinización, el aprovechamiento eficiente de los recursos hídricos, el tratamiento de aguas residuales y las tecnologías de reciclaje y

\footnotetext{
${ }^{25}$ Naciones unidas ha establecido los Objetivos de Desarrollo Sostenible (ODS), también conocidos como Objetivos Mundiales, que son un llamado universal a la adopción de medidas para poner fin a la pobreza, proteger el planeta y garantizar que todas las personas gocen de paz y prosperidad.
} 
reutilización. Sin olvidar apoyar y fortalecer la participación de las comunidades locales en la mejora de la gestión del agua y el saneamiento ${ }^{26}$.

De igual manera, es oportuno tener en cuenta también lo que plantea la CEPAL (2016, p. 44) en el sentido de que las políticas deben reconocer los positivos y necesarios encadenamientos y las sinergias entre temas para hacer más integral y sostenible el desarrollo. Por ejemplo, son bien conocidas las relaciones entre el acceso al agua potable y al saneamiento con las condiciones de salud; la asociación que existe entre la infraestructura para agua y para riego; entre los beneficios de mediano plazo de la educación, y los ingresos de los hogares y la salud; y entre los programas de cuidado social y la autonomía de las mujeres. Esta formulación plantea retos a la mejor secuencia de los programas públicos. En realidad, lo más sinérgico será el tejido que se crea y se activa, que se pueda dar de muchas maneras.

\section{CONCLUSIONES}

Más allá de la reflexión propuesta y de realizar la recopilación in genere de lo expuesto, se desea llamar la atención sobre la oportunidad que presenta el momento histórico que está viviendo Colombia, con el fin de generar propuestas que permitan la construcción de una paz a través de la identificación y atención de necesidades particulares de la población. Con lo dicho entonces tenemos que, la realidad de la ruralidad colombiana respecto del abastecimiento de agua y por ende la garantía del derecho humano al agua, se constituye en una necesidad impostergable que debe asumirse y garantizarse a través de acciones concretar que deben adoptarse de manera urgente. No se puede desconocer que en la actualidad actual se presenta por fuera de los regímenes jurídicos de distribución del líquido diseñados por el Estado: los servicios públicos domiciliarios, los distritos de riego y el derecho ambiental. Es más, es una mezcla de los tres o un punto intermedio entre estos.

Por ello, consideramos que se requiere de una política pública que contemple el suministro de agua en el medio rural con una visión integral, basada en la realidad y no en la tendencia ideal o deontológica que se tenga de la ruralidad. En suma, es de vital importancia que Colombia avance en implementar acciones encaminadas a mejorar las condiciones de vida de las comunidades, en la construcción de un cuerpo normativo y la elaboración de políticas publicas especiales que tengan en cuenta las particularidades de la zona rural del país ${ }^{27}$.

\footnotetext{
${ }^{26}$ Véase en la página web del Programa de las Naciones Unidas para el Desarrollo. Específicamente en el link: $<$ https://goo.gl/YkPcXt>.

${ }^{27}$ Así pues, se pueden generar avances primordiales, si se contara con los siguientes aspectos: una política unificada tanto para la protección del recurso, como para su acceso en la zona rural; la implementación de tecnologías apropiadas y
} 
Por ello, lo dispuesto en el Acuerdo de Paz y su reformulación, si bien se constituye como un avance en la materia, a través del presente artículo nos permitimos proponer como desarrollo en los avances del mismo, la creación, diseño, concertación, promulgación y evaluación de políticas públicas, normas y reglamentación que tenga en cuenta las necesidades de la ruralidad, no dentro de los esquemas de servicios públicos, distritos de riego o el derecho ambiental, sino uno nuevo.

Esta nueva perspectiva debe reunir además de los anteriores elementos, como mínimo que se establezca que los sistemas de distribución sean viables o factibles desde el punto de vista financiero, técnico y operativo para satisfacer las necesidades de las personas que se encuentran a grandes distancias. Adicionalmente, que la distribución del agua posea unos costos acordes a los ingresos per cápita de los habitantes de la zona rural, en cuyo caso, la intervención del Estado se debe generar a partir del respeto de las condiciones tradicionales y culturales de la zona en cuestión, para lo cual, es indispensable que los sistemas de abastecimiento estén acorde con las capacidades técnicas de los habitantes. Es indispensable entonces un nuevo cuerpo jurídico que posibilite los usos múltiples del agua, es decir, “tecnología multipropósito”28 como pueden ser captación de agua lluvia ${ }^{29}$, subterránea y superficial sectorizada por necesidad ${ }^{30}$, entre otros.

El Estado colombiano debe emprender acciones orientadas a asegurar mejores condiciones de vida para la población rural, superando así la pobreza, los problemas de salud, las necesidades de servicios públicos y el acceso adecuado al recurso hídrico necesario para mejorar sus condiciones y promover el desarrollo integral del campo. Por ello entonces, el abastecimiento de agua en el contexto del Acuerdo de Paz además de garantizar varios derechos como el derecho humano al agua, representa un aporte al logro condiciones que garanticen la paz estable y duradera para Colombia.

\section{REFERENCIAS}

COMISIÓN ECONÓMICA PARA AMÉRICA LATINA Y EL CARIBE (CEPAL). Bienes y servicios públicos sociales en la zona rural de Colombia. Brechas y políticas públicas. Juan Carlos

\footnotetext{
alternativas que permitan el acceso al agua, así como las normas necesarias para que esto sea posible. La creación de un sistema de subsidios apropiado a la realidad de la zona rural. Lo cual confirma sin lugar a dudas, la necesidad de contar con un cuerpo normativo e institucional que este avocado único y exclusivamente a las particularidades de la zona rural colombiana.

${ }^{28}$ Temática importante a definir por cuanto las particularidades de cada sector debe ser evaluadas en una nueva opción normativa que recoja el abastecimiento de agua de la zona rural. A manera de ejemplo las obligaciones de micromedición, las estructuras tarifarias, la facturación, desviaciones significativas, índices de agua no contabilizada entre muchos otros.

${ }^{29}$ Desafortunadamente en Colombia se ha considerado que el uso de aguas lluvias está asociado a poblaciones atrasadas y por ende ha venido al desuso. No obstante es una muy buena opción para la solución de los usos múltiples.

${ }^{30}$ Hay que llamar la atención que el RAS 2000, relaciona el nivel de complejidad de los sistemas de distribución de acuerdo al tamaño de la población atendida o su capacidad económica y no por su grado o nivel de tecnológico: como la necesidad de abarcar grandes áreas dispersas y usos múltiples del agua.
} 
Ramírez J., Renata Pardo, Olga Lucía Acosta y Luis Javier Uribe. Naciones Unidasd, CEPAL, Santiago, 2016.

CORPORACIÓN AUTÓNOMA REGIONAL DE CUNDINAMARCA (CAR). Carta Ambiental No. 25. Bogotá D.C., 2010.

CORREA C., Hernán Darío. Acueductos Comunitarios, Patrimonio Público y Movimientos Sociales. Bogotá D.C., 2006. Véase en: <https://goo.gl/cZz7Xp>.

CORTE CONSTITUCIONAL DE COLOMBIA (CCC). Sentencia T-418 de 2010. M.P.: María Victoria Calle Correa.

DEFENSORÍA DEL PUEBLO - COLOMBIA (DP). La Gestión Comunitaria del Pueblo. Bogotá D.C.: Imprenta Nacional, 2013.

DEPARTAMENTO NACIONAL DE PLANEACIÓN - COLOMBIA (DNP). Bases del Plan Nacional de Desarrollo 2014-2018 “Todos por un nuevo país”. Bogotá, 2014.

DEPARTAMENTO NACIONAL DE PLANEACIÓN - COLOMBIA (DNP). Documento CONPES 3344, Lineamientos y Estrategias de Desarrollo Sostenible para los Sectores de Agua, Ambiente y Desarrollo Territorial. Bogotá, 2005.

DINAR, Ariel; KECK, Andrew. Private irrigation investment in Colombia: effects of violence, macroeconomics policy and environmental conditions. World Bank, 1996.

FARAH Q., María Adelaida; PÉREZ C., Edelmira. Mujeres rurales y nueva ruralidad en Colombia, artículo que recoge lo dicho en la ponencia presentada en el II Congreso Iberoamericano de Estudios de Género, en la mesa de trabajo sobre Mujeres rurales y organización. Salta, Argentina, 24-26 de julio de 2003. Bogotá: Cuadernos de Desarrollo Rural, 2004.

GÓMEZ-REY, Andrés; RODRÍGUEZ, Gloria Amparo. El derecho fundamental al agua. Desde el derecho ambiental y los servicios públicos domiciliarios. Colección precedentes jurisprudenciales. Universidad del Rosario, LEGIS, Bogotá, 2013.

INSTITUTO DE HIDROLOGÍA, METEOROLOGÍA Y ESTUDIOS AMBIENTALES DE COLOMBIA (IDEAM). Estudio Nacional del Agua. Bogotá: Imprenta Nacional, 2015.

MINISTERIO DE EDUCACIÓN DE COLOMBIA (MINEDUCACIÓN). Sistema Nacional de Indicadores Educativos para los Niveles de Preescolar, Básica y Media en Colombia. Bogotá D.C., 2014.

ORGANIZACIÓN DE LAS NACIONES UNIDAS PARA LA ALIMENTACIÓN Y LA AGRICULTURA (FAO). Alimentos para la Ciudades. 2009. Véase en: <https://goo.gl/WeLhbV>.

ORGANIZACIÓN MUNDIAL DE LA SALUD (OMS). Fondo De Las Naciones Unidas Para La Infancia. Progresos en materia de saneamiento y agua potable: informe de actualización. 2010. Véase en: <https://goo.gl/2NUCZP>.

RAMÍREZ, Diana Patricia et al. Oportunidades para la conservación de la biodiversidad local, Conectividad Ecológica en la Zona Urbano-Rural, Instituto de Investigación de Recursos Biológicos 
Alexander von Humboldt, Bogotá, D.C., 2008.

RAMÍREZ, Jorge Enrique Torres. Misión para el Diseño de una Estrategia de Reducción de la Pobreza y la Desigualdad. Contrato de Consultoría No. 192-04, 2005.

RED INTERNACIONAL PARA LOS DERECHOS ECONÓMICOS, SOCIALES Y CULTURALES (RED-DESC). Observación general No 15: El derecho al agua (artículos 11 y 12 del Pacto Internacional de Derechos Económicos, Sociales y Culturales). Red-DESC, 2001.

TARQUINO, Inés Restrepo. Usos múltiples del agua, como estrategia para la reducción de la pobreza. Santiago de Cali: Programa Editorial Universidad del Valle, 2010.

Andrés Gómez-Rey Maestría en Derecho Administrativo. Profesor Temporal en la Universidad del Rosario. Email: andres.gomez@urosario.edu.co 